\title{
Calidad de los suelos agrícolas y rentabilidad en cultivos de papa y cereales en la provincia de Chupaca - Junín, 2017
}

\section{Quality of agricultural soils and profitability in potato and cereal crops in the province of Chupaca - Junín, 2017}

\author{
apariona Benavides, L.; Bullón Ames, J.P.; ' García Pariona, L.D. \\ Facultad de Agronomía / Universidad Nacional del Centro del Perú \\ Email: Ipariona@uncp.edu.pe
}

\begin{abstract}
Resumen
La actividad económica, más importante, en la provincia de Chupaca es la agricultura de tipo intensivo, la cual altera la regulación natural de los suelos agrícolas y consecuentemente la pérdida de su calidad, que afecta a la producción y la variación de la rentabilidad de los cultivos. El objetivo de la investigación, fue determinar cómo influye la calidad de los suelos agrícolas en la rentabilidad de los cultivos de papa, maíz y cebada. La clasificación de la calidad de los suelos se obtuvo a partir de una muestra de 25 series de suelos, que fueron analizados en el laboratorio, cuyos resultados permitieron agruparlos en cuatro categorías: Clase A, corresponde a suelos de alta calidad; Clase B, suelos de mediana calidad; Clase $\mathrm{C}$, suelos de baja calidad y; Clase $\mathrm{D}$, que corresponde a suelos pobres. Con respecto a la rentabilidad en el cultivo de papa, se obtuvo una rentabilidad proporcional para suelos de alta y mediana calidad, decreciendo la rentabilidad entre $1 \%$ y $36 \%$ en suelos de baja calidad, con pérdidas económicas en suelos de calidad pobre que requieren una alta fertilización y manejo agronómico de los suelos; en el cultivo de maíz, la rentabilidad es proporcional para suelos de calidad alta, media, baja y pobre calidad de suelo; mientras el cultivo de cebada, tuvo una rentabilidad baja en suelos de mediana, baja y pobre calidad a excepción de los suelos de alta calidad.
\end{abstract}

Palabras clave: actividad agrícola, análisis de suelo, producción agrícola, rentabilidad agrícola, indicadores de calidad agrícola

\begin{abstract}
The most important economic activity in the province of Chupaca is intensive agriculture, which alters the natural regulation of agricultural soils and consequently the loss of their quality, which affects production, and the variation of the profitability of farmer's crops. The objective of the research was to determine how the quality of agricultural soils influences the profitability of potato, corn and barley crops. The classification of soil quality was obtained from a sample of 25 soil series, which were analyzed in the laboratory, whose results allowed grouping them into four categories: Class A corresponding to high quality soils, Class B to medium soils quality, Class C to low quality soils and Class D corresponding to poor soils. With respect to the profitability in potato cultivation, a proportional profitability was obtained for high and medium quality soils, decreasing the profitability between $1 \%$ and $36 \%$ in low quality soils, with economic losses in poor quality soils which require high fertilization and agronomic management of the soils; in the corn crop, the profitability is proportional for high, medium, low quality soils and poor soil quality, while barley cultivation had a low profitability in medium, low and poor quality soils with the exception of high quality soils.
\end{abstract}

Keywords: agricultural activity, soil analysis, agricultural production, agricultural profitability, agricultural quality indicators 


\section{Introducción}

Durante los últimos años, la rentabilidad de los principales cultivos en la provincia de Chupaca ha decrecido en más de $30 \%$, según el MINAG (2016). Este fenómeno se debe principalmente al efecto de la producción intensiva de los cultivos y uso de tecnologías inadecuadas de manejo de suelos; por tanto, la existencia de indicadores de la calidad del suelo, permiten conocer el estado nutricional, comportamiento y utilidad de los suelos agrícolas, teniendo en cuenta el estado de las propiedades físicas, químicas, biológicas en un momento dado (Larson y Pierce, 1994), lo cual forma parte de la interacción entre calidad basada en las propiedades intrínsecas que definen la capacidad productiva del suelo y las características ambientales de sustentabilidad productiva como son la a) Resiliencia y estabilidad, b) Caracterización del sistema de manejo y, c) Identificación de los puntos críticos distribuidos en el agro ecosistema, que permitirá evaluar productividad, rendimiento y rentabilidad de los cultivos, así como mantener el crecimiento de las plantas sin que ocurra degradación ni daños en el medio ambiente, siendo la función principal de proveer un medio para el crecimiento de las plantas (Comité de la Sociedad de la Ciencia del Suelo Americana, 2018); así como, regular y distribuir el flujo del agua en el ambiente y servir como amortiguador de los cambios climáticos.

Como se puede apreciar, este nuevo concepto sustenta, por un lado, las propiedades intrínsecas del suelo y sus interacciones y, por otro, las relaciones del suelo con el medio ambiente y las plantas; sin embargo, la amplitud de conceptos y la especificidad a la que puede ser aplicado, son elementos fundamentales del análisis de las características económicas y divergentes, debido a la situación de la creciente pérdida de calidad del suelo y sus interrelaciones existentes en el manejo del suelo, por la que, se puede establecer reglas de manejo y preservación de este recurso en forma integral, como también depende de los actores sociales involucrados en el manejo del suelo, para determinar su calidad y asegurar la cantidad y calidad para la producción a alimentos.

Sin embargo, la rentabilidad es fluctuante, en la conceptualización económica, donde la rentabilidad como medida del rendimiento en un determinado periodo produce capitales utilizados en el mismo. Esto supone la comparación entre la renta generada y los medios utilizados para obtener producción, lo que permitiría elegir alternativas o la eficiencia del uso de la calidad del suelo mediante la regulación natural de los suelos agrícolas, y no tendríamos suelos vulnerables, dependientes de insumos químicos e intervención antrópica. Márquez (2015), realizó un estudio comparativo de sistemas de manejo de café orgánico en 18 fincas cafetaleras ubicadas en la zona de Turrialba y Orosi, y su impacto sobre la calidad de los suelos, evaluó el efecto de los sistemas de manejo sobre los indicadores de calidad de suelo donde se midieron indicadores químicos, ( $\mathrm{pH}, \mathrm{K}, \mathrm{Ca}, \mathrm{Mg}, \mathrm{P}, \mathrm{Cu}, \mathrm{Fe}, \mathrm{Mn}$, Zn, Acidez, BAS, \% saturación aniónica, carbono activo y carbono mineral), físicos (densidad aparente) y biológicos, (bacterias y hongos, \% de materia orgánica, respiración microbiana, número de lombrices, biomasa de la cobertura y hojarasca). Salomón (2015), observó que existe diferencias significativas entre tratamientos con las variables, densidad aparente, \% de materia orgánica, respiración microbiana, número de lombrices y biomasa de la hojarasca y calculó que el índice de calidad de suelo aditivo (ICSA), fue mayor en el sistema bosque que entre todos los sistemas evaluados y que entre los sistemas de manejo de café estudiados, las mejores condiciones lo presentan los sistemas orgánicos.

Cantú (2007), concluyó que el índice de calidad del suelo, igual a 0,47 , mediante un conjunto mínimo de indicadores conformado por carbono orgánico, $\mathrm{pH}$, saturación de bases, agregados estables en agua, velocidad de infiltración, densidad aparente y el espesor del horizonte, pertenecen a la clase de moderada calidad donde el valor del ICS fue fuertemente influenciado por el indicador carbono orgánico y considerado un atributo, dada su marcada influencia sobre la mayoría de las propiedades del suelo. Carrillo (2015), generó un análisis descriptivo y de diagnóstico de los usos del suelo, que permitió conocer las fortalezas y limitaciones de los métodos que miden la calidad de uso de los suelos y que son empleados como herramientas, en la planificación de cultivos para evitar situaciones de riesgo y que el peligro de los suelos merme la productividad de los cultivos.

Pulido (2014), mencionó que las áreas de pastoreo que ocupan vastas extensiones en el suroeste de España, con más de 4 millones de hectáreas, soportan aproximadamente de 1 de 3 millones de cabezas de ganado, entre cabras ovejas, cerdos y vacas, y aprovechan de manera intensiva sus pastos, lo que ha generado una mala gestión del recurso suelo por el sobrepastoreo, que llegó a provocar la pérdida de la calidad del suelo y afrontar graves pérdidas económicas y daños ambientales. Minaya (2015), en su análisis de la rentabilidad en la producción de papa blanca comercial en las regiones de Huánuco y Lima, concluyó que los niveles de rentabilidad que se pueden obtener a partir de la producción de papa blanca comercial en las regiones de Huánuco y Lima, es a partir del indicador: margen bruto por hectárea, siendo ampliamente superior en Lima, en contraste, con los resultados obtenidos, en la región Huánuco que presenta mayores niveles de riesgo en la producción de papa blanca comercial, pues los cambios que se pueden originar en las variables independientes como la calidad del suelo, afectan en mayor medida la variabilidad del margen bruto por hectárea, ocasionando que esta actividad sea considerada riesgosa por los agricultores. 
En la provincia de Chupaca, de igual manera se evidenció un alto riesgo en la producción y rentabilidad de sus cultivos, por lo que se formuló el siguiente problema: ¿Cómo influye la calidad de los suelos agrícolas en la rentabilidad de los cultivos de papa, maíz y cebada en la provincia de Chupaca-Junín 2017? y; el objetivo general que se planteó fue: Determinar la influencia de la calidad de los suelos agrícolas en la rentabilidad de los cultivos de papa, maíz y cereales en la provincia de Chupaca-Junín.2017; justificándose el estudio, porque permite conocer la calidad de los suelos y la rentabilidad en cada serie de suelos y plantear la distribución adecuada de los cultivos en cada calidad de suelo para obtener mayor rentabilidad.

\section{Materiales y métodos}

\section{Lugar de ejecución}

El contexto de la investigación, comprendió el estudio de 25 series de suelos agrícolas que encuentran a una altitud de $3352 \mathrm{msnm}$, perteneciente a la ecorregión de serranía esteparia. La primera clase, corresponde a suelos aluviales recientes de terrazas bajas del grupo II, con drenaje moderado a pobre y con pendiente casi a nivel y no gravoso; la segunda clase, corresponde a suelos aluviales de terrazas altas del grupo III, que son suelos con drenaje bueno a moderado, de pendiente casi a nivel $\mathrm{u}$ ondulado, moderadamente grueso a muy gravoso y con tres subgrupos: (a) suelos inundables de textura gruesa a media y calcáreo superficial; (c) suelos de textura media a fina y; (e) suelos de textura media a fina y calcáreos moderadamente profundos.

\section{Selección de la población y muestra}

Los suelos agrícolas de la provincia de Chupaca tienen una extensión de 48,917.13 ha. Este estudio se realizó a través de la clasificación de los suelos a partir de muestras representativas de las series de suelos clasificados según el mapa semidetallado de la provincia de Chupaca a $40 \mathrm{~cm}$ de profundidad en una sola etapa durante el año 2018.

La muestra consta de 25 series en estudio con 2083 ha seleccionados de acuerdo a la clasificación preliminar de suelos del valle del Mantaro.

\section{Metodología de la investigación}

La presente investigación, se desarrolló en cuatro etapas de trabajo, según la guía de descripción de suelos de la FAO (2009). La primera etapa, consistió en el acopio de la información secundaria con la revisión bibliográfica, compilación de mapas, elaboración del mapa base, preparación de la guía de evaluación y calificación de suelos; la segunda etapa, fue de campo, comprendió: a) La delimitación de los suelos en estudio. b) Caracterización y zonificación de 25 series de suelos en una extensión de 2083 ha. y, c) Caracterización y toma de muestra de los suelos en estudio; la tercera etapa, consistió en determinar los indicadores físicos-químicos de los suelos como la textura mediante el método de Bouyoucos, la estructura a través del ensayo de campo, color comparando pequeñas cantidades de suelo con la Tabla Munsell, densidad aparente con el método del cilindro, $\mathrm{pH}$ método del potenciómetro, determinación de la materia orgánica con el método de Walkley y Black, Nitrógeno, método de MicroKjendahl, fósforo, método de Olsen y potasio, método de Peach, en el Laboratorio de Análisis de Suelos de la Facultad de Agronomía de la Universidad Nacional del Centro del Perú. En la cuarta etapa de gabinete, se ordenaron y clasificaron los datos de costos, precios proporcionados por la Oficina de Estadística de la Agencia Agraria de Chupaca del Ministerio de Agricultura para sistematizar y hallar la rentabilidad de los cultivos en las diferentes series en estudio.

\section{Análisis de datos}

Para evaluar la rentabilidad se usó información de producción, precios y rendimiento de los cultivos de papa, maíz y cebada. Se realizó el análisis de varianza respectivo, con un nivel de 0,05 \% de probabilidad. La prueba de la Normalidad, según el test de normalidad de Kolmogorov - Smirnov, para ponderar la calidad del suelo y la rentabilidad de los cultivos Los datos obtenidos de los resultados de análisis de suelos fueron clasificados y sistematizados a través del software: Word, Excel y SPSS 23 para estandarizar y validar los datos.

\section{Registro de variables}

La variable calidad de suelos comprendió, el estudio de 25 series, Ma-s (Mantaro superficial), Ma - s/B (Mantaro superficial pendiente de 2 a $7 \%$ ), Ch (Chupaca) Ch/C Chupaca (con pendiente de 7 a $15 \%$ ), Cp (Chucampe), Az (Azana), Az-g (Azana Gravoso), Az/ g-D (Azana gravoso con pendiente de 15 a $25 \%$ ), Cu (Cunas), $\mathrm{Cu} / \mathrm{B}$ (Cunas con pendiente de 2 a $7 \%$ ), $\mathrm{Cm}$ (Carmen), Cm/B (Carmen con pendiente de $2^{\mathrm{a}} 7 \%$ ), $\mathrm{Cm} / \mathrm{C}$ (Carmen con pendiente de 7 a $15 \%$ ), Ha (Huayao), Ha/B (Huayao con pendiente de 2 a $7 \%$ ), Ha/C (Huayao con pendiente de 7 a $15 \%$ ), Ha/g (Huayao gravoso), $\mathrm{Ha} / \mathrm{g}-\mathrm{B}$ (Huayao gravoso con pendiente de 2 a $7 \%$ ) $\mathrm{Ha} / \mathrm{g}-\mathrm{C}$ (Huayao gravoso con pendiente de 7 a $15 \%$ ), Ha - pe ( Huayao pesado), Ha-pe/B (Huayao pesado con pendiente de 2 a $7 \%$ ) Ha-pe/D (Huayao pesado con pendiente de 15 a $25 \%$ ), Ha-pe-ca (Huayao pesado calcáreo), Ha-pe- ca/B (Huayao pesado calcáreo), Ha-pe- ca/g-D (Huayao pesado calcáreo con pendiente de 7 a $15 \%$ ).

La variable de rentabilidad, se estructuró en base a los costos de producción, rendimiento y precios de los cultivos en estudio. 


\section{Prueba de hipótesis estadística}

Los datos obtenidos de los resultados de análisis de suelos fueron clasificados y sistematizados a través del software: Word, Excel y SPSS 23 para estandarizar y validar los datos.

\section{Resultados}

\section{Resultados de la clasificación de los suelos por su calidad}

En la Tabla 1, se muestra los resultados de la clasificación de la calidad de los suelos de 25 series en estudio, el cual se realizó comparando las propiedades físico-químicas y biológicas de suelos de las series en estudio. Las unidades básicas están constituidas por suelos que son similares en las principales características del perfil, agrupándose en función a la textura, $\mathrm{pH}$, contenido de materia orgánica, CIC, N, P y K, de acuerdo a los indicadores de calidad según Obade \& Cantú (2007).

Tabla 1

Clasificación de la calidad de suelos

\begin{tabular}{|c|c|c|c|c|}
\hline Clases & $\begin{array}{l}\text { Clasificación } \\
\text { de la Calidad }\end{array}$ & Series & Área & $\begin{array}{l}\text { Cantidad } \\
\text { de Series }\end{array}$ \\
\hline A & Alta & $\begin{array}{l}\mathrm{Ma}-\mathrm{s} / \mathrm{B}, \mathrm{Ch} / \mathrm{C}, \mathrm{Cp}, \\
\mathrm{Az} / \mathrm{g}-\mathrm{D}, \mathrm{Cu}, \mathrm{Cu} / \mathrm{B}, \\
\mathrm{Ha}, \mathrm{Ha} / \mathrm{g}-\mathrm{B}, \mathrm{Ha}- \\
\mathrm{Pe} / \mathrm{D}, \mathrm{Ha}-\mathrm{pe} . \mathrm{ca} / \mathrm{B}\end{array}$ & 545 & 7 \\
\hline $\mathrm{B}$ & Mediana & $\begin{array}{l}\mathrm{Az}, \mathrm{Az} / \mathrm{g}, \mathrm{Cm}, \\
\mathrm{Cm} / \mathrm{B}, \mathrm{Ha} / \mathrm{g}, \\
\mathrm{Ha} / \mathrm{g}-\mathrm{C}, \mathrm{Ha}-\mathrm{pe}, \\
\text { Ha-pe/B, Ha-pe-ca, } \\
\text { Ha-pe-ca/g-D }\end{array}$ & 728 & 12 \\
\hline $\mathrm{C}$ & Baja & $\mathrm{Ha}, \mathrm{Ha} / \mathrm{C}, \mathrm{Ma}-\mathrm{s}$ & 133 & 4 \\
\hline \multirow[t]{2}{*}{$\mathrm{D}$} & Pobre & $\mathrm{Ch}, \mathrm{Cm} / \mathrm{C}$ & 677 & 2 \\
\hline & & Total & 2082 & 25 \\
\hline
\end{tabular}

Nota: Representa la clasificación de los suelos agrícolas de la provincia de Chupaca en cuatro clases de calidades de suelo: A alta, B media, C baja y D pobre, con 12 series de suelos que tienen calidad media.

En la Figura 1, se muestra el dendrograma, en el cual se observa que los suelos de calidad media, son las que tiene diferencias entre las series en función a sus propiedades físicas y químicas dentro de la misma localidad, mostrando mayor concentración de series. Estos grupos, que se forman, crean observaciones en cada caso con niveles de similitud, donde el nivel de similitud se muestra en el eje vertical y las diferentes observaciones se especifican en el eje horizontal. Se observa; así mismo, que se han formado cuatro grupos de suelos, los mismos que han sido clasificados según la FAO utilizando los indicadores de calidad de suelo, como son la textura, estructura, densidad aparente, $\mathrm{pH}$, materia orgánica y elementos nutritivos como nitrógeno total, fósforo disponible y potasio disponible, planteados por Doran y Parkin (1994).

\section{Figura 1}

Dendrograma del análisis de agrupamiento en cuatro clases de la calidad de los suelos A, B, C y D de acuerdo a sus características físicas y químicas.

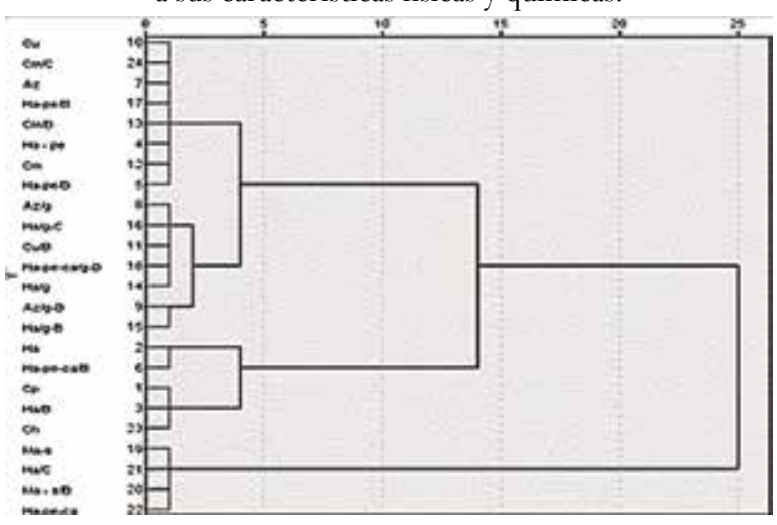

\section{Resultados de la rentabilidad en el cultivo de papa}

Los resultados de rentabilidad del cultivo de papa en la provincia de Chupaca, se evaluaron teniendo en consideración los costos de producción constante de S/. 5514 . 24 soles y, la producción promedio por año y los precios de venta de $0.35,0.50$ y 0.60 céntimos, precios en chacra, considerando la estacionalidad de producto y la regulación del mercado.

En la Tabla 2, prueba de T para la rentabilidad del cultivo de papa, se observa que existe diferencia estadística significativa en suelos de alta y mediana calidad cuando los precios de venta son menores o igual a $\mathrm{S} / .0 .35$ céntimos de Sol, la rentabilidad baja, asimismo en los suelos pobres genera pérdidas económicas para el agricultor; por esa razón, para obtener rentabilidad, los precios deben estar por encima de S/.0,50 céntimos de Sol el kilogramo y ser sembrados en suelos de alta calidad para asegurar el retorno económico de la inversión y tener rentabilidad (Vargas, 2016).

Tabla 2

Prueba de T para rentabilidad del cultivo de papa.

\begin{tabular}{lcccc}
\hline \multicolumn{5}{c}{ Valor de prueba $=\mathbf{0}$} \\
\hline & $\mathbf{t}$ & gl & $\begin{array}{c}\text { Sig. } \\
\text { (Bilateral) }\end{array}$ & $\begin{array}{c}\text { Diferencia } \\
\text { de medias }\end{array}$ \\
\hline Rentabilidad 1 & -1.958 & 24 & 0.062 & 5.48 \\
Rentabilidad 2 & 10.304 & 24 & 0.000 & 21.45 \\
Rentabilidad3 & 23.598 & 24 & 0.000 & 38.47
\end{tabular}

Nota: Existe significación estadística para la rentabilidad 1; es decir, cuando los precios bajan a S/.0.35 céntimos de Sol, en chacra, se obtiene menor rentabilidad en suelos de mediana y baja calidad.

La Figura 2, muestra que el mayor \% de rentabilidad se obtiene en las series cuya calidad de suelo agrícola es de la clase B o calidad media, esto se debe a que las propiedades físicas, químicas y biológicas favorecen al suelo con una mejor capacidad retentiva de agua, así como, una buena 
estructura que son favorables por ser suelos aluviales de terrazas altas, que probablemente se ven favorecidas por la ligera pendiente; la rentabilidad en suelos de alta calidad es menor, esto se debe a que las series que conforman este grupo, como las series $\mathrm{Ch} / \mathrm{C}, \mathrm{Cp}, \mathrm{Ha}, \mathrm{Ha}-\mathrm{pe}, \mathrm{Ha} / \mathrm{B}, \mathrm{Ha}-$ pe/D y Ha-pe-ca/B son suelos de textura fina, arcillosos y pesados que van a influir en el desarrollo de los tubérculos disminuyendo la producción y, consecuentemente, la rentabilidad del cultivo va a ser menor en comparación con los suelos de calidad media; en suelos de baja calidad y calidad pobre la rentabilidad es menor, por lo tanto, se debe recomendar un manejo de cultivos adicionando materia orgánica al suelo (Bolaños, Tapia, Soto \& Filho, 2012).

Figura 2

Se observa el mayor porcentaje de rentabilidad del cultivo de papa en suelos de mediana calidad en la provincia de Chupaca.

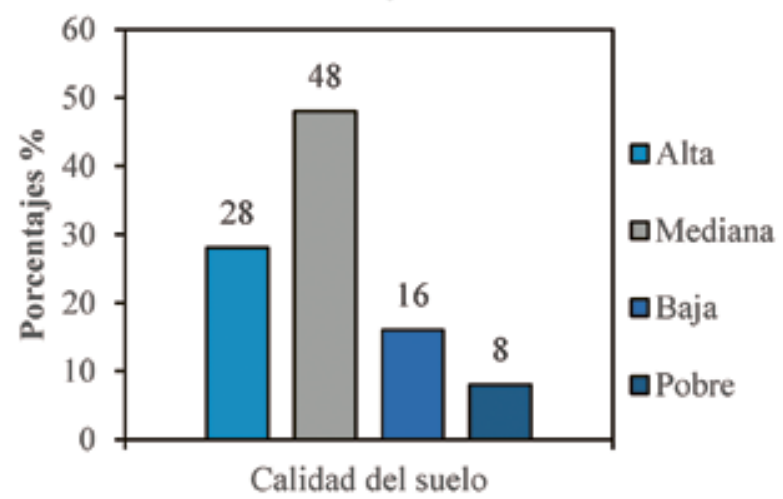

Resultados de la rentabilidad en el cultivo de maíz

En la Tabla 3, de las comparaciones múltiples para la rentabilidad en el cultivo de maíz, se observa que existe significación estadística cuando se compara la rentabilidad entre suelos de calidad alta con suelos de calidad pobre, suelos de calidad media con suelos de calidad baja y pobre, suelos de calidad baja con suelos de calidad media, pobre y alta, mientras que los suelos de calidad pobre muestra diferencias altamente significativas; es decir, que la rentabilidad de los cultivos está directamente relacionada con la calidad de suelos para la producción de maíz.

Tabla 3

Comparación múltiple para la rentabilidad del cultivo de maíz.

\begin{tabular}{ccccc}
\hline Calidad de suelo & N & Media & $\begin{array}{c}\text { Desviación } \\
\text { típica }\end{array}$ & $\begin{array}{c}\text { Error } \\
\text { típico de la } \\
\text { media }\end{array}$ \\
Alta/Rentabilidad & 7 & 64.89 & 2.315 & 0.875 \\
Media/Rentabilidad & 12 & 54.64 & 1.804 & 0.521 \\
Baja/rentabilidad & 4 & 51.16 & 2.153 & 1.076 \\
Pobre/Rentabilidad & 2 & 55.91 & 16.033 & 11.337 \\
\hline
\end{tabular}

Nota: Existe significación estadística para la rentabilidad 1; es decir, cuando los precios bajan a S/.0.35 céntimos de Sol, en chacra, se obtiene menor rentabilidad en suelos de mediana y baja calidad.
La Figura 3, muestra que existe una mayor rentabilidad del cultivo de maíz para suelos de alta calidad siendo proporcional para suelos de media y baja calidad; asimismo, se observa que los rendimientos de maíz en suelos pobres son bajos y según las pruebas de normalidad para la rentabilidad en el cultivo de maíz no existe significación estadística (0.037) y se acepta la hipótesis de que la rentabilidad en el cultivo de maíz depende de la calidad de los suelos y de la producción.

\section{Figura 3}

Diagrama de cajas para ver las características importantes de la dispersión y simetría de la rentabilidad del cultivo de maíz con respecto a la calidad de los suelos.

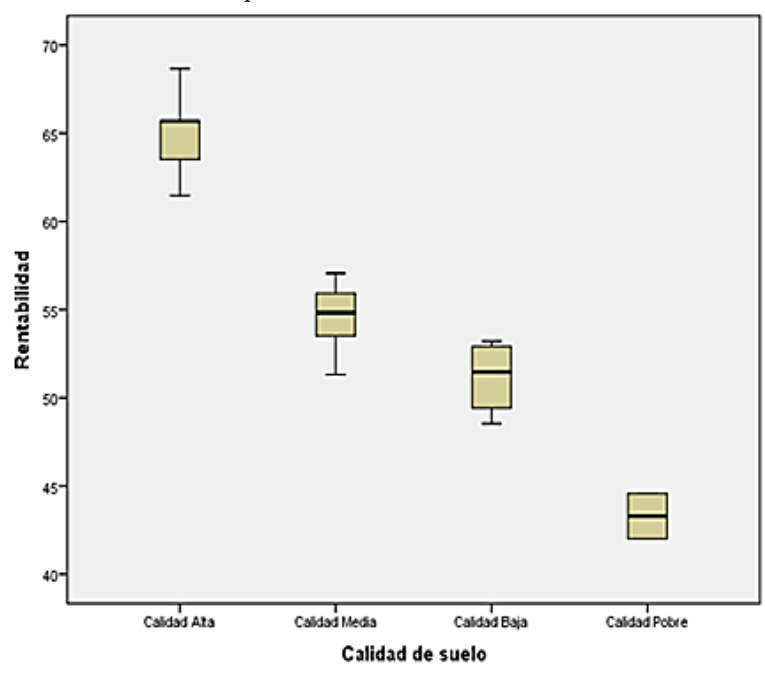

Resultados de la rentabilidad en el cultivo de cebada

La rentabilidad del cultivo de cebada al precio de venta de $\mathrm{S} / .1 .80$ soles, es mayor en las series $\mathrm{Ha}, \mathrm{Ha} / \mathrm{B}$ y Hape, pero si los cultivos de cebada se siembran en suelos de mediana calidad, la rentabilidad no se justifica porque va de $1 \%$ a $9 \%$ siendo un cultivo que muchas veces se siembra con fines de forraje para los animales, porque la producción y la rentabilidad de grano seco es muy bajo; asimismo, en los suelos de baja calidad como la serie Ha. pe-ca, la rentabilidad es alta, ello se puede atribuir a la presencia de material calcáreo en el suelo, el cual estaría influenciando en la mayor producción y rentabilidad de este cultivo y no así por el precio, Se recomendaría instalar el cultivo de cebada en suelos de calidad pobre porque se obtendrían rendimientos medios.

\section{Discusión}

\section{Resultados de la clasificación de los suelos por su calidad}

Las clases de suelos, agrupadas por su calidad, permiten hacer un análisis de las características físicas químicas de los suelos y segundo ver la importancia que tienen con respecto a la producción de los cultivos. Los suelos 
de clase A, de alta calidad, conformado por las series : Ch/C, Cp, Ha, Ha-pe, Ha/B, Ha-pe/D y Ha-pe-ca/B representan el $26 \%$ del área en estudio con 545 ha, estos suelos se han caracterizado por presentar un $\mathrm{pH}$ neutro que indica que los elementos nutritivos, como nitrógeno, fósforo y potasio se encuentran en sus formas disponibles para la absorción inmediata por la planta; así como, presentan una capacidad de intercambio catiónico (CIC) alta en la capa arable, lo cual le permite retener los cationes adsorbidos como nutrientes para las plantas (Gonzáles, 2006). El incremento en la producción puede deberse a la fertilidad del suelo por la presencia de los elementos nutritivos disponibles; sin embargo, la capa arable de estos suelos presenta una textura media a fina, por la presencia de arcillas finas, que se expanden y contraen de acuerdo al régimen de humedad Ustic, caracterizada en la zona, donde está parcial o totalmente seco por más de 90 días acumulados durante el año, el cual influye en la formación de los tubérculos de la papa, más no así en los cultivos de maíz y cebada que tienen un sistema radicular fibroso.

Los suelos de Clase B, de mediana calidad, comprenden un área de 728 ha, representando el $35 \%$ del área en estudio, superficie mayor respecto a las clases de suelos de calidad alta, baja y pobre, conformado por las series. $\mathrm{Az}, \mathrm{Az} / \mathrm{g}, \mathrm{Az} / \mathrm{g}-\mathrm{D}, \mathrm{Cu}, \mathrm{Cu} / \mathrm{B}, \mathrm{Cm}, \mathrm{Cm} / \mathrm{B}, \mathrm{Ha} / \mathrm{g}, \mathrm{Ha} /$ g-B, Ha/g-C, Ha-pe/B y Ha-pe-ca/g-D, la principal característica de estos suelos, es mantener una buena capacidad productiva debido a las altas concentraciones de nitrógeno, fósforo y potasio, complementadas con una textura franca del suelo que le permite tener una buena capacidad retentiva de agua y aire; sin embargo, las limitaciones de estos suelos se deben a la presencia de gravosidad y a las ligeras pendientes que generan escorrentía superficial del agua e interfieren en el manejo agronómico de los cultivos de papa, maíz y cebada, debido a la mala disposición de surcos. Masaguer (2013), menciona que estos problemas pueden ser controlados con el empleo de tecnologías modernas; así como, mejorando la estructura del suelo con la incorporación de materia orgánica, porque al carecer de una buena estructura, presentan un bajo porcentaje de poros, siendo ligeramente compactos, producidos por la pérdida de vegetación y falta de materia orgánica. No obstante, estos suelos son muy importantes para la producción agrícola.

Los suelos de clase $\mathrm{C}$ de baja calidad, están conformados por 4 series: Ma-s, Ma-s/B, Ha/C y Hape-ca, en una extensión de 133 ha y representan el 6\% del área total. Estos suelos tienen severas limitaciones en su fertilidad natural, tales como profundidad efectiva muy baja, contenido de materia orgánica menor al $1 \%$, textura muy arcillosa o muy arenosa, baja capacidad retentiva de agua, o excesiva retención, entre otros. Por ello, su producción agropecuaria es ineficiente y solo pueden ser aprovechados para suelos de reforestación o para protección o bosques nativos (Martínez, 2001).

Finalmente, la clase D son suelos de pobre calidad, conformado por 677 has de las series: Ch y $\mathrm{Cm} / \mathrm{C}$ y representan el $33 \%$ de los suelos de la provincia de Chupaca, son suelos compactados, muestran capas duras (claypans) y costras, estos suelos son marginales para la explotación agrícola y deben ser utilizados para la conservación de flora y fauna silvestre (Gómez, 2016), a pesar de ello, los agricultores lo utilizan para la producción agrícola, conllevando más adelante a la degradación de los suelos, por ser una explotación irracional de los cultivos no teniendo en consideración las características del suelo, que les puede permitir obtener mayor rentabilidad, siendo conveniente adaptar un manejo alternativo de tecnologías, apropiadas en la que se destacan la disminución de laboreo en la preparación del suelo, para no ser perturbado, proteger permanentemente la superficie del suelo con cobertura vegetal viva o muerta, incrementar el contenido de materia orgánica con mayor aporte de estiércol o compost de buena calidad combinados con el uso de fertilizantes químicos en cantidades adecuadas.

Estas acciones deben complementarse entre sí, para crear un suelo que tenga una mayor capacidad de absorción del agua de lluvia, y mantener un suelo biológicamente rico (Medina, 2012), de esta manera se reduciría la erosión y mantendría un suministro de alimento continuo a las plantas y, consecuentemente, tener suelos de alta calidad, para soportar un máximo de producción en cantidad y calidad; también, equilibrar algunas características muy diferenciadas que se debe tener en cuenta para hacer uso y manejo de las diferentes series en estudio.

\section{Resultados de la rentabilidad en el cultivo de papa}

La rentabilidad del cultivo de papa, se evaluó teniendo en cuenta tres precios de venta del producto en chacra, S/.0.35, S/.0.50 y S/.0.60 céntimos de Sol, la estacionalidad del producto y la regulación del mercado, contrastando con la calidad de los suelos, en suelos de alta calidad la rentabilidad es proporcional a los precios, pero las series $\mathrm{Ha}$-pe, $\mathrm{Ch} / \mathrm{C}$ y Ha-pe/D la rentabilidad decrece drásticamente entre 1 y $9 \%$ cuando la papa se vende a S/.0.35 céntimos de Sol siendo más óptimo vender la papa a un precio mayor de S/.0.50 o S/.0.60 céntimos de Sol, para que la rentabilidad pueda llegar a $50 \%$. En los suelos de mediana calidad cuando los precios de venta son bajos a S/.0.30 céntimos de Sol, hay pérdidas económicas que pueden llegar hasta $-17 \%$; a excepto en la serie $\mathrm{Cm}$, donde puede haber una ligera rentabilidad del 2 
$\%$, pero cuando se vende a S/.0.50 y S/.0.60 céntimos de Sol la rentabilidad puede llegar hasta el $41 \%$ en la serie Ha-pe-ca/G-D. En los suelos de baja calidad no existe rentabilidad, sino pérdidas económicas o mínimas ganancias, estas variaciones, no necesariamente van a estar relacionadas con la producción, sino también, con la variación de los precios en el mercado aseverado por Astier, Maass y Echevers (2015), lo que significa que, además de influir en la producción por la calidad del suelo, estos resultados se van a modificar por el precio de los productos en el mercado, a pesar que se pueda plantear un mejor manejo agronómico, aspecto que se debe tener en cuenta al evaluar rentabilidad de los cultivos, García (2002).

Los suelos pobres tienen bajos niveles de NPK, con una estructura angular y subangular que impide una buena aireación y retención del agua, el suelo de alta calidad tiene una rentabilidad proporcional a los precios, pero puede descender drásticamente entre 1 y $9 \%$ cuando la papa se vende a S/.0.35 céntimos de Sol siendo óptimo comercializar la papa cuando los precios sean igual a $\mathrm{S} / .0 .50$ o $\mathrm{S} / .0 .60$ céntimos de Sol o lo superen, y la rentabilidad puede llegar a 50 $\%$. En los suelos de mediana calidad cuando los precios de venta son bajos a S/.0.30 céntimos de Sol, hay pérdidas económicas que pueden llegar hasta -17 $\%$ a excepto en la serie $\mathrm{Cm}$ donde puede haber una ligera rentabilidad del $2 \%$ que cuando se vende a $\mathrm{S} / .0 .50$ y S/.0.60 céntimos de Sol donde la rentabilidad puede llegar hasta el $41 \%$; en los suelos de baja calidad, no existe rentabilidad sino pérdidas económicas o mínimas ganancias, lo que significa que esos suelos tienen que tener un mejor manejo agronómico y obtener mayor rentabilidad; con respecto a los suelos pobres, se puede apreciar que en la serie Ch la rentabilidad es ligeramente mayor a la serie $\mathrm{Cm} / \mathrm{c}$, aunque no supera a los suelos de mayor calidad ni de mediana calidad; sin embargo, cuando la papa se comercializa con los tres precios planteados se obtiene rentabilidad pero, cuando el precio es bajo esa rentabilidad también es baja.

Según la prueba de $\mathrm{T}$ para la variable rentabilidad en el cultivo de papa muestra que existe significación estadística para la rentabilidad 1; por esta razón, para obtener rentabilidad los precios deben estar por encima de S/.0,50 céntimos de Sol el kilogramo y ser sembrados en suelos de alta calidad para asegurar el retorno económico. Los costos de producción que se consideró para el cultivo de papa fueron de S/.5 514.24 soles que se calcularon en base a los costos que el productor incurre en la provincia de Chupaca, y que se distribuyeron en costos variables; es decir, aquellos costos que dependen del nivel de producción y costos fijos que no dependen del nivel de produc- ción, de los cuales el $84 \%$ corresponde a costos variables y el $16 \%$ a costos fijos. Estos costos se distribuyeron en tres etapas durante el proceso de producción (periodo vegetativo, que para la papa viene a ser aproximadamente seis meses) los cuales son: siembra, labores agrícolas y cosecha.

De los costos variables, el $56 \%$ corresponde a la siembra, el cual se efectúa en los dos primeros meses, aproximadamente, el $31 \%$ para labores agrícolas que se efectúa entre el tercer y quinto mes, aproximadamente y el $13 \%$ para la cosecha efectuado en el sexto mes, generalmente.

\section{Resultados de la rentabilidad en el cultivo de maíz}

La rentabilidad del cultivo de maíz, es mayor en suelos de calidad alta y media, teniendo una relación directa con la rentabilidad del cultivo; no obstante, se puede apreciar que en suelos de pobre calidad la rentabilidad es ligeramente superior porque el sistema radicular de la planta es fibroso y el suelo, al tener una estructura de tipo franco arenoso, las raíces se estabilizan mejor en comparación con los suelos de calidad baja.

Las comparaciones múltiples para la rentabilidad no muestran significación estadística cuando se compara la rentabilidad entre suelos de calidad alta con suelos de calidad pobre, suelos de calidad media con suelos de calidad baja y pobre, suelos de calidad baja con suelos de calidad media y pobre y alta, mientras que los suelos de calidad pobre muestra diferencias altamente significativas con respeto a la rentabilidad de los cultivos que está directamente relacionada con la calidad de suelos para la producción de maíz (Sánchez, 2001).

\section{Resultados de la rentabilidad en el cultivo de cebada}

La rentabilidad del cultivo de cebada, teniendo en cuenta precio de venta a $\mathrm{S} / .1 .80$ soles, muestra que existe rentabilidad en las series $\mathrm{Ha}, \mathrm{Ha} / \mathrm{B}$ y Ha-pe con los mayores porcentajes de rentabilidad; pero si los cultivos de cebada se siembran en suelos mediana calidad, la rentabilidad no se justifica porque varía de $1 \%$ a $9 \%$ siendo un cultivo que muchas veces se siembra con fines de forraje para los animales, considerando que en producción en grano y la rentabilidad de grano es muy bajo; asimismo, en los suelos de baja calidad como la serie Ha.pe-ca, la rentabilidad es alta, pudiendose atribuir a la presencia de calcáreos, lo que haría que la producción de este cultivo se incremente y aumente la rentabilidad. Se recomendaría instalar en suelos de pobre calidad porque sus rendimientos son medios, mientras que si se siembra en suelos de calidad media las propiedades físicas, químicas y biológicas favorecen al suelo con una mejor capacidad retentiva de agua; así como, una buena estructura que son favorables especialmente para suelos aluviales de terrazas 
altas como la que se presenta en la provincia de Chupaca, (Gregorich et al., 1994), y que probablemente se vean favorecidas por la ligera pendiente, mientras que si se siembra la cebada en suelos de calidad alta la rentabilidad es menor, esto se debería a que las series que conforman este grupo como las series $\mathrm{Ch} / \mathrm{C}, \mathrm{Cp}$, Ha, Ha-pe, Ha/B, Ha-pe/D y Ha-pe-ca/B son suelos de textura fina, arcillosos, y pesados que van a compactar el sistema radicular de los cultivos que estarían sometidos a la expansión y contracción del suelo perjudicando el desarrollo de las raíces y consecuentemente disminuiría la producción, como lo manifestó Vallejo (2013) y, por tanto, la rentabilidad del cultivo va a ser menor en comparación con los suelos de calidad media, en suelos de baja calidad y calidad pobre la rentabilidad es menor por lo tanto se debe recomendar un manejo de cultivos adicionando materia orgánica al suelo (Cabrera, 2012).

\section{Conclusiones}

- Se clasificó la calidad de los suelos según protocolo de la FAO (2009), y se agrupó en cuatro clases: Clase A, $7 \%$ de suelos de alta calidad que comprende las series Ch/C, Cp, Ha, Ha-pe,Ha/B, Ha-pe/D y Ha-pe-ca/B. Clase B, $12 \%$ de suelos de calidad media conformada por las series Az, Az/g, Az/g-D, $\mathrm{Cu}, \mathrm{Cu} / \mathrm{B}, \mathrm{Cm}, \mathrm{Cm} / \mathrm{B}, \mathrm{Ha} / \mathrm{g}, \mathrm{Ha} / \mathrm{g}-\mathrm{B}, \mathrm{Ha} / \mathrm{g}-\mathrm{C}$, Ha- pe/B y Ha-pe-ca/g-D. Clase C, $4 \%$ de suelos de calidad baja que corresponde a las series Ma-s, $\mathrm{Ma}$-s/B, Ha/C y Ha-pe-ca. Clase D, 2 \% de suelos de calidad pobre conformado por las series $\mathrm{Ch}$ y $\mathrm{Cm} / \mathrm{C}$.

- Con respecto a la calidad del suelo y la rentabilidad del cultivo de papa, no existe diferencia estadística significativa, estimando la rentabilidad con precios de venta de $\mathrm{S} / .0 .35, \mathrm{~S} / .0 .50 \mathrm{y} \mathrm{S} / .0 .60$ céntimos de Sol y la estacionalidad del producto, a excepto en las series Ha-pe, Ch/C y Ha-pe/D cuya rentabilidad decreció de $1 \%$ a $36 \%$.

- La calidad del suelo y la rentabilidad en el cultivo de maíz es proporcional para cada clase de calidad de suelo.

- Con respecto al cultivo de cebada, la rentabilidad es baja, a excepto en suelos de baja calidad

- Los suelos de mediana calidad no tienen restricciones ni limitaciones que alteran la calidad de los suelos y se puede establecer cualquiera de los cultivos estudiados obteniendo una rentabilidad que justifica los costos de producción.

- Los agricultores carecen de conocimientos sobre el manejo de la calidad de suelo y eficientes prácticas de conservación de suelos para maximizar la rentabilidad de los cultivos de papa, maíz y cebada en la provincia de Chupaca.

\section{Referencias bibliográficas}

Americana, C. d. (2018). Facultad de Agronomía.

Astier Calderón.Marta; Maass Moreno, Manuel; Echevers Barra, Jorge. (2015). Derivación de Indicadores de calidad de suelos en el contexto de la agricultura sustentable. Agrociencia, 316. doi: Texoco - México

Bolaños, N. Tapia, A. y Filhho (2012). Efecto de diferentes sistemas de manejo sobre la calidad del suelo, en fincas cafetaleras de la zona de Turrialba y Orosi. Intersedes, 85-105.

Brañez. (2014). Análisis de la diversidad de suelos y series en la provincia de Almería. España: Universidad Politécnica de Madrid.

Cabrera. (2015). Meso Fauna Edáfica. Indicador Biológico de la calidad del suelo. Tesis, En la Fundación Rufford, Cuba.

Cantú. (2007). Evaluación de la calidad de suelos mediante el uso de indicadores e índices. Tesis, Universidad Nacional de Río, Argentina.

Carlos. Percy. Ramírez. Cayro, Ávila Rojas, Lucio. (2013). Modelo para medir la rentabilidad de los cereales altoandinos en el departamento de Puno. Caso quinua. Revista de Investigación Alto Andina, 15(1), 1-199.

Carrillo. (2015). Efectos de la capacidad de uso en la calidad de los suelos en la denominación de origen Montilla- Moreiles. Universidad de Córdova, Argentina.

Daniel, W. (1990). Estadística inferencial. México: Interamericana.

De Paul Obade, L. (2016). A standardized soil qualyti index for diverse field conditions. Science of the total environment, 541-424-434.

De Paul Obade, V. \& Lal Cantú. (2007). Evaluación de la calidad de suelos mediante el uso de indicadores e indices. Tesis, Universidad Nacional de Río, Argentina.

FAO. (2009). Guía de la clasificación de la calidad de los suelos agrícolas.

García, E. (2002). Estudio de costo y rentabilidad para el manejo de los tipos forestales Roble-Raulí-Caihue y Siempre verde. Precordillera Andina. Proyecto FDI.

Gómez. (2016). Caracterízación de los suelos de usos agrícolas e industrial de la Comarca de Molina de Segura (Murcia) para una planificación racional del territorio. Tesis, Universidad de Valencia, España. 
Gonzáles. (2006). Metodología, formulación y aplicación de un índice de calidad de suelos con fines agrícolas para Castilla la Mancha, morfología, mineralogía y génesis de los suelos. Tesis, Universidad Autónoma de Madrid, Madrid.

Gregorich. (1994). Towards a mínimun data set to assess soil organic matter quality in agricultural soil. Soil Science, 367-385.

Hernández, H. (1997). Estudio de productividad y rentabilidad de cultivos agrícolas. Manejo sustentable de cultivos.

Márquez. (s.f.). Indicadores para evaluar la sustentabilidad en las fincas cafetaleras en Quillabamba. Tesis, Universidad Nacional Agraria La Molina, Lima Perú.

Martínez, H. (2001). Protección de zonas agroecológicas productoras de papas nativas.

Masaguer. (2013). Valoración de indicadores de calidad de suelos para el diseño e implementación de tecnologías de manejo de Luvisoles de la zona norte de la provincia de Tunas. Tesis, Universidad Politécnica de Madrid, Madrid España.

Medina. (2012). Caracterización bioquímica y microbiólogica de un suelo de pradera de Dactilis glomerata y Medicago sativa bajo diferentes proporciones de siembra. Tesis, Universidad de Salamanca, España.

Minaya. (2015). Análisis de la rentabilidad en la producción de papa blanca en las regiones de Huánuco y Lima. Tesis, Huánuco.

Partkin, D. y. (1994). Defining and assessing soil qualyti. Soil Science Society of América.

Pierce, L. y. (1994). Conservation and enhancement of soil quality. Bangkok, 175.

Púlido. (2014). Indicadores de calidad del suelo en áreas de pastoreo. Tesis, España.

Salomón Barrezueta, Unda, Paz Gonzáles, Antonio, Chabla, Julio. (2015). Determinación de indicadores para calidad de suelos cultivados con cacao en provincia de el Oro-Ecuador. Tesis, Universidad Técnica de Machala, Ecuador.

Sánchez. (2001). La rentabilidad en los cultivos de papa y cereales. Lima-Perú: Aliaga S.A.

SIEA. (2017). Estadíticos. Obtenido de http://www. grade.org.pe/biblioteca/otros recursos de información/estadísticas-y-datos.
Vallejo. (2013). Importancia y utilidad de la evaluación de la calidad de suelos mediante el componente microbiano: Experiencias en sistemas silvopastoriles. Universidad Distrital Francisco José de Caldas, Bogotá-Colombia.

Vargas Oviedo, J. E. (2016). Análisis de la rentabilidad de la Tara. Tesis, Apurimac.

UNESCO. (2016). Objetivos del Milenio. 